\title{
Access to 2,6-Dipropargylated BODIPYs as "Clickable" Congeners of Pyrromethene-567 Dye: Photostability and Synthetic Versatility
}

\author{
Clara Uriel, Ana M. Gómez,* Enrique García Martínez de la Hidalga, Jorge Bañuelos,* \\ Inmaculada Garcia-Moreno, and J. Cristobal López*
}

Cite This: Org. Lett. 2021, 23, 6801-6806

Read Online

\section{ACCESS | Lill Metrics \& More | 回 Article Recommendations ｜（） Supporting Information}

ABSTRACT: Hitherto unreported 2,6-dipropargyl-1,3,5,7-tetramethyl BODIPYs can be efficiently prepared by a Nicholas reaction/decomplexation protocol from 1,3,5,7-tetramethyl BODIPYs. The title compounds, which improve the BODIPY photostability by retaining their inherent photophysical and photochemical properties, can be engaged in efficient copper(I)catalyzed azide-alkyne cycloaddition (CuAAC) "click-type" reactions with azido derivatives to provide all-BODIPY-triads or conjugated BODIPYs.

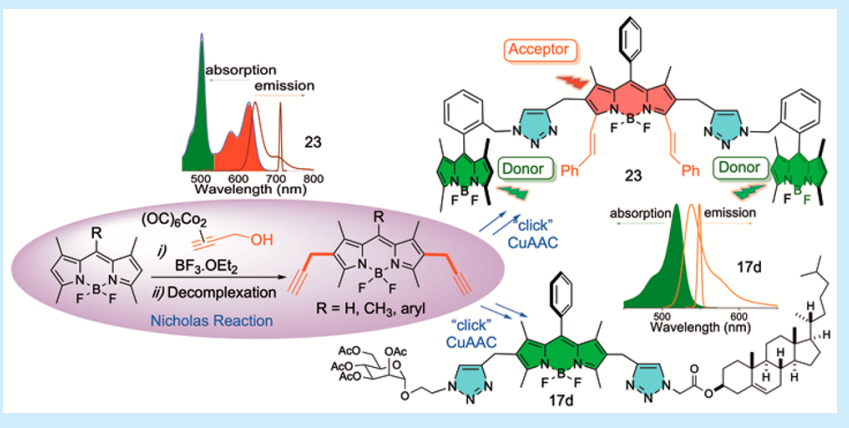

R ODIPY (4,4'-difluoro-4-bora-3a,4a-diaza-s-indacene) dyes, e.g., 1 (Figure 1), have recently established themselves as one of the most appealing families among the arsenal of small-molecule fluorophores. ${ }^{2}$ BODIPYs have found ample applications in diverse areas ranging from biology to material sciences, e.g., photodynamic therapy, ${ }^{3}$ labeling of biomolecules, ${ }^{4}$ tunable laser dyes, ${ }^{5}$ organic photovoltaics, photosensitizers, and components in organic light-emitting diodes (OLEDs) ${ }^{6}$ and light harvesting systems. ${ }^{7}$ The reasons for their success can be credited to their remarkable photophysical properties, which include high fluorescence quantum yields and photostability, and large molar absorptivity. However, it is probably their chemical flexibility and stability that makes them the fluorophores of choice in a variety of applications. Thus, it has been shown that structure modifications can fine-tune their photophysical, physical, and chemical properties, and this has converted the pursuit of synthetic methods to incorporate a variety of functionalities in the BODIPY core in an active area of research. For instance, selected functionalization of the skeleton can induce bathochromic $^{8}$ or hypsochromic ${ }^{9}$ shifts in their absorption and emission bands, water-solubility, ${ }^{10}$ and modification of their photostability. ${ }^{11}$ Regarding the latter, a variety of structural modifications aimed at improving the photochemical stability of commercially available pyrromethene 567 (PM567) laser dye 2 (Figure 1), ${ }^{12}$ frequently used as an internal reference for fluorescence quantum yields, ${ }^{13}$ have been examined, particularly at positions C-8, C-2, C-6, and at boron (Figure 1). ${ }^{14}$

With these considerations in mind, we envisioned that hitherto unreported BODIPY 3 (Figure 1), a "clickable" analogue of PM567 (vide infra), could be an attractive laserdye building-block with potential implications in some of the

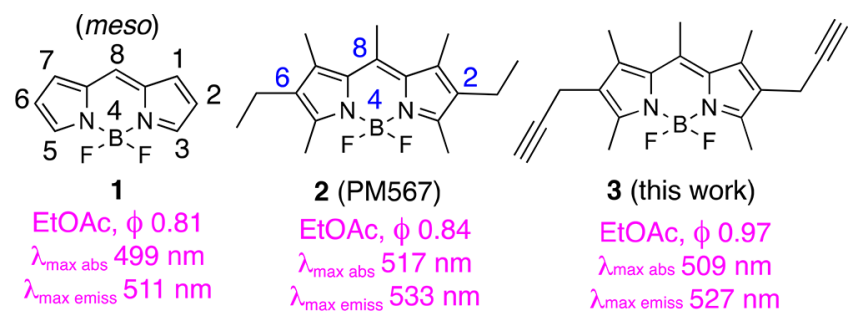

Figure 1. BODIPY 1, pyrromethene 567 (PM567) 2, and 2,6dipropargyl BODIPY 3, and spectroscopic data.

aforementioned applications. In this manuscript, we describe the synthesis, and some photophysical and photostability studies, of 2,6-dipropargyl BODIPY 3 , and some of its congeners. In addition, we have investigated their use as fluorescent tags and as components in light-harvesting BODIPY triads.

Initial propargylation studies were performed on 1,3,5,7tetramethyl BODIPYs with $O$-propargyl trichloroacetimidate and related agents under a variety of reaction conditions. ${ }^{15}$ However, although in some instances, the desired 2,6dipropargyl derivatives could be obtained, the transformation-in our hands - proved to be unreliable. Consequently, as a method to incorporate the propargyl substituent(s) to the

Received: July 16, 2021

Published: August 17, 2021 
BODIPY core, we selected the Nicholas reaction (promoted by a Lewis acid) in which an electrophilic propargylic cation, stabilized by a cobalt complex, reacts with a nucleophile. ${ }^{16,17}$ An additional decomplexation step is then necessary to unveil the desired alkyne moiety. Thus, overall the Nicholas reaction occurs with high regioselectivity at the propargylic position and has a wide scope in terms of reactive nucleophiles.

As the initial BODIPY to test the Nicholas reaction, we selected 1,3,5,7,8-pentamethyl BODIPY 4 (see Scheme 1).

Scheme 1. Nicholas Reaction of BODIPY 4 Leading to 2,6Dipropargyl BODIPY 3, by Iodine-Promoted Decobaltation of Synthetic Intermediate 6

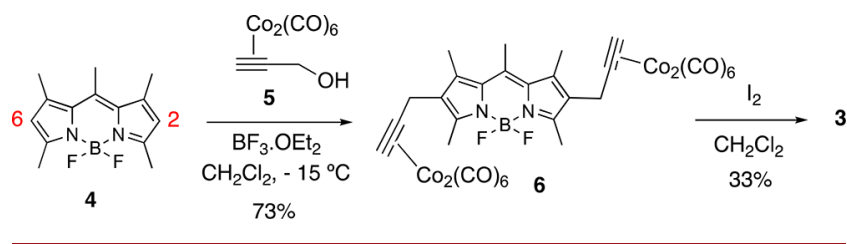

Accordingly, treatment of 4 with the dicobalthexacarbonyl complex of propargyl alcohol (5) in $\mathrm{CH}_{2} \mathrm{Cl}_{2}$, at $-15{ }^{\circ} \mathrm{C}$, in the presence of $\mathrm{BF}_{3} \cdot \mathrm{OEt}_{2}$ ( 0.5 equiv), yielded alkynyl-cobalt intermediate 6 , which was decobaltated upon treatment with iodine in $\mathrm{CH}_{2} \mathrm{Cl}_{2}$ to yield 2,6-dipropargyl BODIPY 3 (see Scheme 1). Thus, according to the well-known reactivity of 1,3,5,7-tetramethyl BODIPYs, ${ }^{18}$ an electrophilic aromatic substitution $\left(S_{E} A r\right)$ had taken place at positions C-2 and C-6 of the BODIPY core with the cobalt-stabilized propargyl cation arising from 5 .

To evaluate the scope of the transformation, we next extended our studies to 1,3,5,7-tetramethyl BODIPYs $7 \mathbf{a}-7 \mathbf{c}$, differing in the substituents at the meso-position (Figure 2).

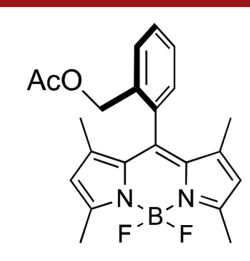

$7 a$

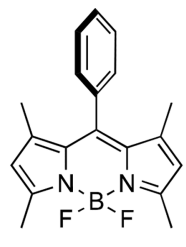

7b

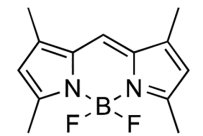

7c
Figure 2. BODIPY derivatives $7 \mathbf{a}-7 \mathrm{c}$.

In keeping with the reaction conditions employed with BODIPY 4 (Scheme 1), tetramethyl-BODIPYs $7 a-7 c$ were treated with 5 under the agency of $\mathrm{BF}_{3} \cdot \mathrm{OEt}_{2}$ ( 0.5 equiv) in $\mathrm{CH}_{2} \mathrm{Cl}_{2}$, to furnish (after decobaltation) 2,6-dipropargyl BODIPYs 10a-10c (see Scheme 2). Thus, upon treatment of BODIPYs $7 a-7 c$ with 2.2 equiv of 5 , the corresponding dipropargylcobalt-BODIPY intermediates $\mathbf{8 a - 8 c}$ could be

Scheme 2. Synthesis of 2,6-Dipropargyl and 2-Propargyl BODIPY Derivatives 10a-10c and 11a-11c, Respectively, by Nicholas Reaction/Decomplexation of BODIPYs $7 a-7 c$

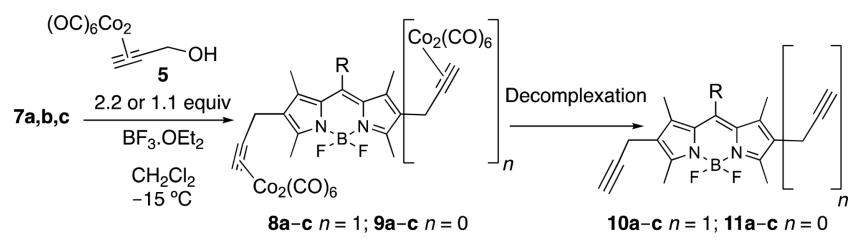

obtained in good to excellent yields (Scheme 2 and Figure 3). Decomplexation of the latter to lead to 2,6-dipropargyl

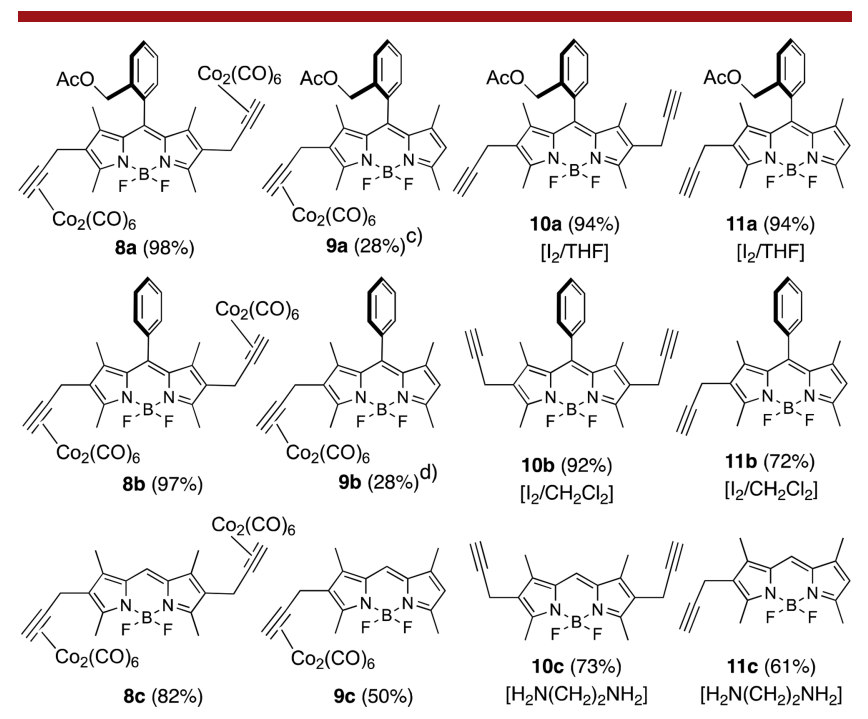

Figure 3. Monopropargyl and dipropargyl BODIPYs 11a-11c and $9 a-9 c$, respectively, along with their intermediate dicobalt-propargyl derivatives $\mathbf{8 a}-\mathbf{8 c}$ and $\mathbf{1 0 a}-\mathbf{1 0 c}$, obtained from BODIPYs $7 \mathbf{a}-\mathbf{7 c}$. Yields and decomplexation methods are included.

BODIPYs 10a-10c, however, demanded some optimization of the reaction conditions. Thus, whereas iodine in THF or $\mathrm{CH}_{2} \mathrm{Cl}_{2}$ worked well with meso-aryl derivatives $\mathbf{8 a}$ and $\mathbf{8 b}$, to furnish dipropargyl-BODIPYs 10a and 10b (Figure 3), 1,2ethylenediamine in $\mathrm{THF},{ }^{19}$ had to be used with the more labile BODIPY derivative $\mathbf{8 c}$, to produce BODIPY $\mathbf{1 0 c}$ (Figure 3 ). In addition, in order to assess the possibility of obtaining synthetically valuable monosubstituted propargyl BODIPYs, i.e., 11a-11c (Scheme 2), the use of limited amounts of 5 (1.1 equiv) in the Nicholas reaction was also explored. Under these conditions, moderate amounts of monosubstituted propargyldicobalt-BODIPY intermediates $\mathbf{9 a - 9 c}$ could be obtained, from which access to monopropargylated BODIPYs 11a-11c could be obtained upon decobaltation (see Scheme 2 and Figure 3).

To obtain 2,6-dipropargyl-BODIPYs with improved photophysical properties, BODIPYs 3 and $\mathbf{1 0 b}$ were transformed to $\mathrm{B}(\mathrm{CN})_{2}$-dipropargyl BODIPYs 12 and 13 , respectively, by treatment with TMSCN in $\mathrm{CH}_{2} \mathrm{Cl}_{2}$, in good to excellent yields (see Figure 4). ${ }^{20}$

The propargylation of the methylene units grafted at position 2 and/or 6 of the BODIPY core does not alter its inherent photophysical properties (see Table $S 1$ in the Supporting Information). Thus, all of the propargylated BODIPY derivatives displayed strong absorption (505-515 $\mathrm{nm}$ with $\varepsilon_{\max }>6 \times 10^{4} \mathrm{M}^{-1} \mathrm{~cm}^{-1}$ ) and fluorescence (520-530

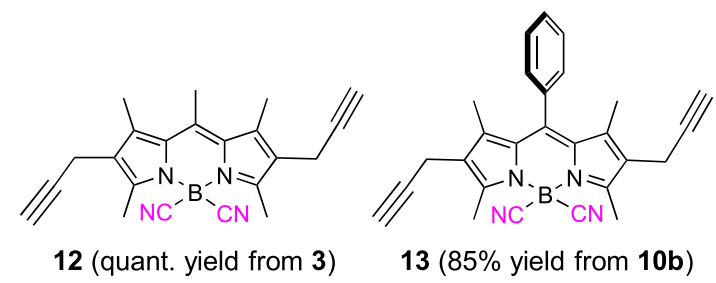

Figure 4. $\mathrm{B}(\mathrm{CN})_{2}$-BODIPYs 12 and 13. 
$\mathrm{nm}$ with $\phi>60 \%$ ) bands (see Figure S1 in the Supporting Information). Nevertheless, some structural factors exerting control on the emission behavior under soft (fluorescence) and hard (laser) irradiation conditions could be drawn out (see Table S1): (i) 2,6-dipropargylated BODIPYs emit more efficiently than their monopropargylated counterparts, e.g., $10 \mathrm{~b}$ vs 11b; I the presence of an 8-alkyl group improves the emission efficiency when compared to that of BODIPYs with 8-phenyl groups, e.g. 3 vs $10 \mathrm{~b}$; (iii) $\mathrm{B}(\mathrm{CN})_{2}$-BODIPYs emit more efficiently than $\mathrm{BF}_{2}-\mathrm{BODIPYs}$ with the same substitution pattern, e.g., 13 vs $10 \mathrm{~b}$. Consequently, dye 12 fulfilling all these structural constraints achieves a fluorescence quantum yield of $100 \%$ and a laser efficiency of $61 \%$ (Table S1). The significant enhancement of the emission efficiency correlates with a lowering of the nonradiative probability by reducing (or avoiding) processes such as charge separation within the pyrroles in the asymmetric BODIPYs, small rotational motion of the 8-phenyl moiety and electronic rearrangement within the dipyrrin framework in cyanoBODIPYs (Table S1). In good agreement, the photostability toward prolonged laser irradiation matches the aforementioned photophysical trends becoming as the most photostable those derivatives based on $\mathrm{B}(\mathrm{CN})_{2}$-dipropargyl BODIPYs (12 and 13) (Figure 5).

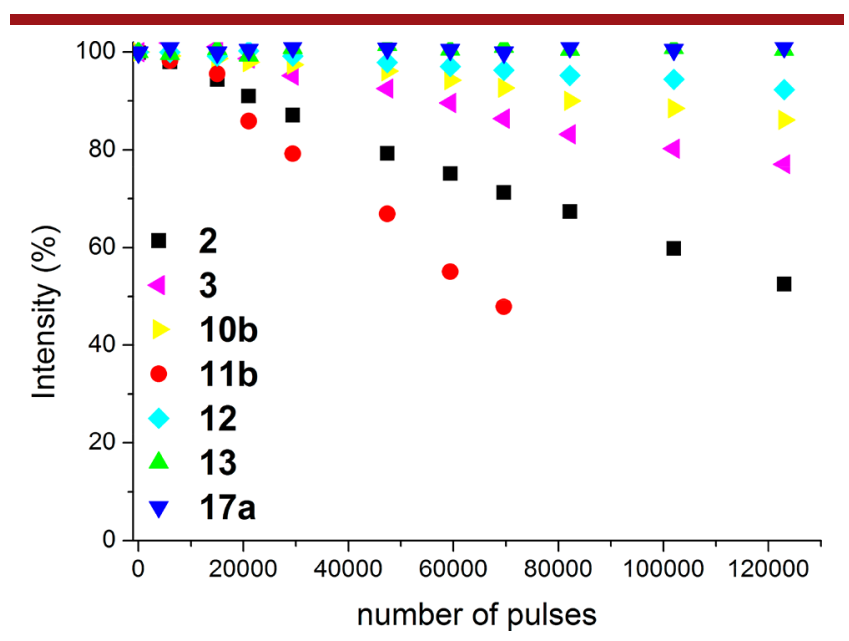

Figure 5. Normalized laser-induced photostability of commercial PM567 (2) and its congeners $3,10 \mathbf{b}, 11 \mathbf{b}, 12,13$, and 17a (vide infra). Optically matched solutions were used.

In order to further illustrate the usefulness of the "clickable"21 2,6-dipropargyl BODIPYs, we have conducted two additional studies with implications in, at least, two of the aforementioned relevant research areas involving BODIPYs: (i) the conjugation to biomolecules and (ii) the preparation of light harvesting systems.

Regarding the first topic, we have investigated the click, copper(I)-catalyzed azide-alkyne cycloaddition (CuAAC), ${ }^{22}$ reaction of dialkynyl BODIPY $10 \mathrm{~b}$, with some biologically relevant compounds. Our exploratory experiments on the $\mathrm{CuAAC}$ reaction of $\mathbf{1 0 b}$ were performed with benzyl azide (14) in the presence of $\mathrm{CuSO}_{4}$ and sodium ascorbate in a glass seal tube $\left(65{ }^{\circ} \mathrm{C}\right)$, leading to bis-1,2,3-triazolyl BODIPY derivative $17 \mathrm{a}$ in $95 \%$ yield ( 3 equiv azido derivatives, $65^{\circ} \mathrm{C}$, Figure 6). We next tested the reactions of $\mathbf{1 0 b}$ with azidocholesteryl derivative 15 and 1-ethylene-2-azido $\alpha$-D-mannopyranosyl glycoside 16. To our satisfaction, both reacted well with $10 \mathrm{~b}$ under the aforementioned experimental conditions,

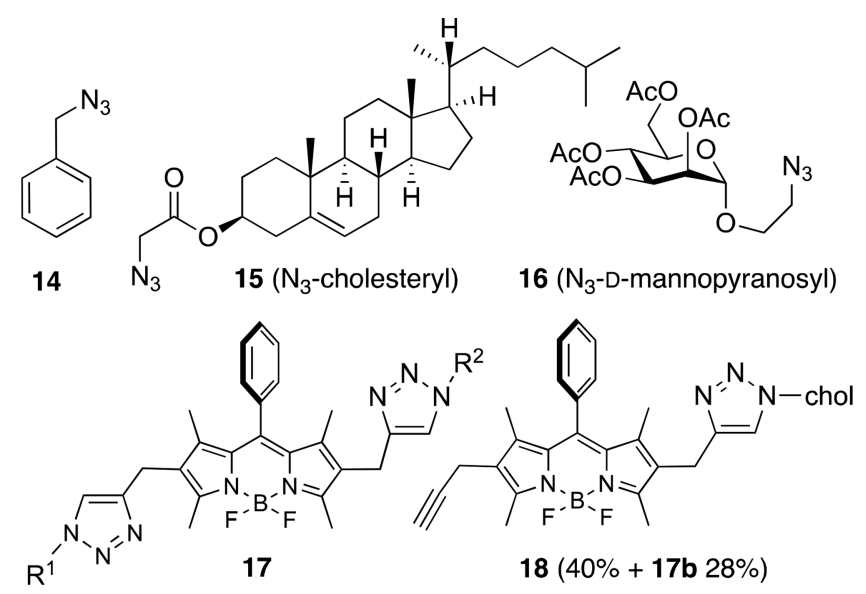

a $\mathrm{R}^{1}=\mathrm{R}^{2}=\mathrm{Bn}(95 \%)$

b $R^{1}=R^{2}=$ cholesteryl $(75 \%)$

c $R^{1}=R^{2}=D-$ mannopyranosyl $(92 \%)$

d $R^{1}=D$-mannopyranosyl; $R^{2}=$ cholesteryl $(78 \%$ from 18$)$

Figure 6. CuAAC-mediated "click" conjugation of BODIPY $10 \mathrm{~b}$. Access to BODIPY-cholesteryl and -Dmannopyranosyl adducts. Sodium ascorbate, $\mathrm{CuSO}_{4}$, glass seal tube, $65{ }^{\circ} \mathrm{C}$.

and yielded bis-1,2,3-triazolyl-cholesteryl and bis-1,2,3-triazolyl-D-mannopyranosyl derivatives $\mathbf{1 7 b}$ and $\mathbf{1 7 c}$, in yields of $75 \%$ and $92 \%$, respectively.

The click reaction of $\mathbf{1 0 b}$ with a limited amount of $\mathbf{1 5}$ (0.9 equiv) allowed the preparation monocholesteryl derivative 18 [40\% yield, along with $17 \mathrm{~b}$ ( $28 \%$ yield)], which, upon a second CuAAC reaction with the azido-mannoside derivative 16 led to the cholesteryl-sugar-BODIPY derivative $17 \mathrm{~d}$ in $78 \%$ yield (Figure 6). This strategy leads to highly photostable BODIPY-tagged biomolecules with highly efficient emission (fluorescence and laser efficiency of $\sim 80 \%$ and $57 \%$, respectively, for $\mathbf{1 7 b} \mathbf{- 1 7} \mathbf{d}$ in Table $S 2$ in the Supporting Information), retaining the BODIPY fine spectroscopic signatures after grafting carbohydrate and/or cholesteryl moieties on its core (see Figure S2). In fact, the presence of the triazolyl moieties, rather than the propargyl groups, confers additional photostability to the ensuing BODIPYs (17a vs $10 \mathbf{b}$ in Figure 5).

Finally, the modular use of dipropargyl BODIPYs 10a and $\mathbf{1 0 b}$, in conjunction with azido-BODIPYs $20^{23}$ and 21 , made possible the CuAAC-mediated assembly of isomeric allBODIPY triads 22a,b and $23,^{24,25}$ where the donor (D) and acceptor (A) roles occupy alternate locations (i.e., A-D-A and D-A-D, respectively, Figure 7). Distyryl BODIPYs 19 and 21 were uneventfully obtained by Knoevenagel condensation of propargyl BODIPY $\mathbf{1 0 b}$ and azido-BODIPY 20, with benzaldehyde in DMF. According to that, the CuAAC reactions of dipropargyl BODIPYs $10 \mathrm{a}$ and $10 \mathrm{~b}$ with azidoBODIPY 21, under the reaction conditions depicted in Figure 7, produced all-BODIPY triads 22 a and 22 b, in $89 \%$ and $60 \%$ yield, respectively, where the donor-role concurs with the central BODIPY unit. Conversely, the CuAAC reaction of dipropargyl-BODIPY 19 with azido-BODIPY 20 led to BODIPY triad 23 (56\% yield), where the central BODIPYunit plays the role of energy acceptor.

This synthetic methodology enables the assessment of the energy donor/acceptor ratio ( $1 / 2$ in $22 b$ and $2 / 1$ in 23 ) dependence on the photophysical properties of these allBODIPY multichromophores. The absorption profile features 
<smiles>C#CCc1c(C)c(C(c2ccccc2)c2c(CC#C)c(C)c(/C=C/c3ccccc3)n2P)n(P)c1/C=C/c1ccccc1</smiles><smiles>Cc1ccccc1C(c1c(C)cc(C)n1P)c1c(C)cc(C)n1P</smiles><smiles>Cc1ccccc1C(c1c(C)cc(/C=C/c2ccccc2)n1P)c1c(C)cc(/C=C/c2ccccc2)n1P</smiles>

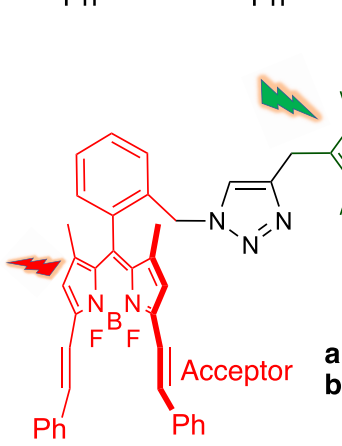<smiles>C1=CC=C1</smiles>

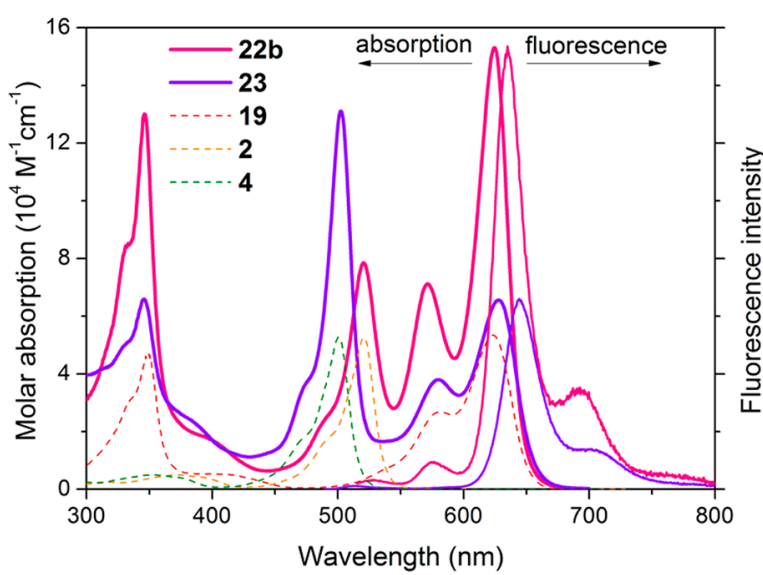

Figure 8. Absorption and normalized fluorescence (dashed, upon excitation $490 \mathrm{~nm}$ ) spectra of the all-BODIPY based cassettes 22b and 23 in diluted ethyl acetate solutions $(\mu \mathrm{M})$. The corresponding absorption spectra (filled) of the free building blocks ( 2 in yellow, 4 in green, and 19 in red) are also added.

molecular cassettes, such as broadband absorption, because of electronic isolation between the building blocks, and efficient EET (almost no sign of emission from the energy donors), which is attributable to the short donor/acceptor distance imposed by their covalent linkage. These structural factors allow one to achieve bright and long-lasting emission at red spectral window, even under drastic pumping conditions.

In summary, we have developed a concise entry to previously unreported 2,6-dipropargyl BODIPYs, which makes use of the Nicholas propargylation reaction. This transformation occurs as an electrophilic aromatic substitution $\left(S_{\mathrm{E}} \mathrm{Ar}\right)$ at the dipyrrin framework by the Nicholas' stabilized dicobalthexacarbonyl propargyl cation. The ensuing 2,6dipropargyl BODIPYs, which displayed improved photostability compared with the parent PM567 dye, can be engaged in highly efficient click azido-alkyne cycloadditions with either two units of the same (bio)molecule or, in a sequential manner, with two different azido-containing (bio)molecules. ${ }^{26}$ The usefulness of these new derivatives has been demonstrated as fluorescent tags, and as modular components in the assembly of all-BODIPY triads. In addition, the application of the Nicholas propargylation reaction to different BODIPY substrates devoid of methyl substituents is under consideration in our laboratory, and the results will be reported in due course.

\section{ASSOCIATED CONTENT}

\section{Supporting Information}

The Supporting Information is available free of charge at https://pubs.acs.org/doi/10.1021/acs.orglett.1c02380.

Experimental and computational procedures, copies of NMR spectra, photophysical data, absorption and fluorescence spectra, simulated absorption spectra and molecular orbitals (PDF)

\section{AUTHOR INFORMATION}

\section{Corresponding Authors}

Ana M. Gómez - Instituto de Química Orgánica General, IQOG-CSIC, 28006 Madrid, Spain; orcid.org/00000002-8703-3360; Email: ana.gomez@csic.es 
Jorge Bañuelos - Departamento de Química Física, Universidad del Pais Vasco-EHU, 48080 Bilbao, Spain; (ㄱ) orcid.org/0000-0002-8444-4383;

Email: jorge.banuelos@ehu.eus

J. Cristobal López - Instituto de Química Orgánica General, IQOG-CSIC, 28006 Madrid, Spain; (i) orcid.org/00000003-0370-4727; Email: jc.lopez@csic.es

\section{Authors}

Clara Uriel - Instituto de Química Orgánica General, IQOGCSIC, 28006 Madrid, Spain

Enrique García Martínez de la Hidalga - Departamento de Química Física, Universidad del Pais Vasco-EHU, 48080 Bilbao, Spain

Inmaculada Garcia-Moreno - Instituto de Química-Física "Rocasolano", CSIC, 28006 Madrid, Spain

Complete contact information is available at: https://pubs.acs.org/10.1021/acs.orglett.1c02380

\section{Author Contributions}

The manuscript was written through contributions of all authors. All authors have given approval to the final version of the manuscript.

Notes

The authors declare no competing financial interest.

\section{ACKNOWLEDGMENTS}

We gratefully acknowledge the Spanish Ministerio de Ciencia e Innovación for financial support (Project Nos. RTI2018094862-B-100, MAT2017-83856-C3-1-P and 3-P; PiD2020$114755 \mathrm{~GB}-\mathrm{C} 31$ and $-\mathrm{C} 33$ ) and the Gobierno Vasco (GV) (Project No. IT912-16) for financial support. We are indebted to Ms. Marina Rodríguez (IQOG-CSIC) for skillful technical support. E.G.M.H. thanks GV for his predoctoral fellowship.

\section{REFERENCES}

(1) (a) Loudet, A.; Burgess, K. BODIPYs and their derivatives: Syntheses and Spectroscopic properties. Chem. Rev. 2007, 107, 48914932. (b) Kolemen, S.; Akkaya, E. Reaction-based BODIPY probes for selective bio-imaging. Coord. Chem. Rev. 2018, 354, 121-134.

(2) (a) Lu, H.; Mack, J.; Yang, Y.; Shen, Z. Structural modification strategies for the rational design of red/NIR region BODIPYs. Chem. Soc. Rev. 2014, 43, 4778-4823. (b) Kowada, T.; Maeda, H.; Kikuchi, K. BODIPY-based probes for the fluorescence imaging of biomolecules in living cells. Chem. Soc. Rev. 2015, 44, 4953-4972.

(3) (a) Kamkaew, A.; Lim, S. H.; Lee, H. B.; Kiew, L. V.; Chung, L. Y.; Burgess, K. BODIPY dyes in photodynamic therapy. Chem. Soc. Rev. 2013, 42, 77-88. (b) Gorbe, M.; Costero, A. M.; Sancenon, F.; Martínez-Mañez, R.; Ballesteros-Cillero, R.; Ochando, L. E.; Chulvi, K.; Gotor, R.; Gil, S. Halogen-containing BODIPY derivatives for photodynamic therapy. Dyes Pigm. 2019, 160, 198-207.

(4) (a) Boens, N.; Leen, V.; Dehaen, W. Fluorescent indicators based on BODIPY. Chem. Soc. Rev. 2012, 41, 1130-1172. (b) Wang, W.; Lorion, M. M.; Martinazzoli, O.; Ackermann, L. BODIPY Peptide labeling by late-stage $\mathrm{C}\left(\mathrm{sp}^{3}\right)-\mathrm{H}$ activation. Angew. Chem., Int. Ed. 2018, 57, 10554-10558.

(5) Banuelos, J.; Martin, V.; Gomez-Duran, C. F. A.; Cordoba, I. J. A.; Pena-Cabrera, E.; Garcia-Moreno, I.; Costela, A.; Perez-Ojeda, M. E.; Arbeloa, T.; Arbeloa, I. L. New 8-amino-BODIPY derivatives: Surpassing laser dyes at blue-edge wavelengths. Chem. - Eur. J. 2011, $17,7261-7270$.

(6) Zampetti, A.; Minotto, A.; Squeo, B. M.; Gregoriou, V. G.; Allard, S.; Scherf, U.; Chochos, C. L.; Cacialli, F. Highly efficient solid-state near-infrared organic light-emitting diodes incorporating A-
D-A dyes based on $\alpha, \beta$-substituted BODIPY moieties. Sci. Rep. 2017, 7, 1611 .

(7) Bozdemir, O. A.; Erbas-Cakmak, S.; Ekiz, O. O.; Dana, A.; Akkaya, E. U. Towards unimolecular luminiscent solar concentrators: BODIPY-based dendritic energy-transfer cascade with panchromatic absorption and monochromatized emission. Angew. Chem., Int. Ed. 2011, 50, 10907-10912.

(8) Wang, J.; Boens, N.; Jiao, L.; Hao, E. Aromatic [b]-fused BODIPY dyes as promising near-infrared dyes. Org. Biomol. Chem. 2020, 18, 4135-4156.

(9) Gomez-Duran, C. F. A.; Garcia-Moreno, I.; Costela, A.; Martin, V.; Sastre, R.; Banuelos, J.; Lopez Arbeloa, F.; Lopez Arbeloa, I.; Pena-Cabrera, E. 8-Propargylamino-BODIPY: Unprecedented blueemitting pyrromethene dye. Synthesis, photophysics and laser properties. Chem. Commun. 2010, 46, 5103-5105.

(10) Kand, D.; Liu, P.; Navarro, M. X.; Fischer, L. J.; Rousso-Noori, L.; Friedmann-Morvinski, D.; Winter, A. H.; Miller, E. W.; Weinstain, R. Water-soluble BODIPY photocages with tunable cellular localization. J. Am. Chem. Soc. 2020, 142, 4970-4974.

(11) Zhang, X.; Xiao, Y.; Qi, J.; Qu, J.; Kim, B.; Yue, X.; Belfield, K. D. Long-wavelenght, photostable, two-photon excitable BODIPY fluorophores readily modifiable for molecular probes. J. Org. Chem. 2013, 78, 9153-9160.

(12) Pavlopoulos, T. G.; Boyer, J. H.; Shah, M.; Thangaraj, K.; Soong, M.-L. Appl. Opt. 1990, 29, 3885-3886.

(13) Valiev, R. R.; Cherepanov, V. N.; Nasibullin, R. T.; Sundholm, D.; Kurten, T. Calculating rate constants for intersystem crossing and internal conversion in the Franck-Condon and Herzberg-Teller approximations. Phys. Chem. Chem. Phys. 2019, 21, 18495-18500.

(14) Thorat, K. G.; Kamble, P.; Ray, A. K.; Sekar, N. Novel pyrromethene dyes with $\mathrm{N}$-ethyl carbazole at the meso position: A comprehensive photophysical, lasing, photostability and TD-DFT study. Phys. Chem. Chem. Phys. 2015, 17, 17221-17236.

(15) (a) Li, C.; Wang, J. Lewis acid catalyzed propargylation of arenes with $O$-propargyl trichloroacetimidates: Synthesis of 1,3diarylpropynes. J. Org. Chem. 2007, 72, 7431-7434. (b) BarrocaAubry, N.; Benchekroun, M.; Gomes, F.; Bonnaffé, D. pMethoxybenzyl-N-phenyl-2,2,2-trifluoroacetimidate: a versatile reagent form mild acid catalyzed etherification. Tetrahedron Lett. 2013, 54, 5118-5121.

(16) Nicholas, K. M. A forty year odyssey in metallo-organic chemistry. J. Org. Chem. 2015, 80, 6943-6950.

(17) (a) Nicholas, K. M. Chemistry and synthetic utility of cobaltcomplexed propargyl cations. Acc. Chem. Res. 1987, 20, 207-214. (b) Díaz, D. D.; Betancort, J. M.; Martín, V. S. The Nicholas reaction: a powerful tool for the stereoselective synthesis of bioactive compounds. Synlett 2007, 2007, 0343-0359.

(18) (a) Boens, N.; Verbelen, B.; Ortiz, M. J.; Jiao, L.; Dehaen, W. Synthesis of BODIPY dyes through postfunctionalization of the boron dipyrromethene core. Coord. Chem. Rev. 2019, 399, 213024. (b) Boens, N.; Verbelen, B.; Dehaen, W. Postfunctionalization of the BODIPY core: Synthesis and spectroscopy. Eur. J. Org. Chem. 2015, 2015, 6577-6595.

(19) Sugihara, T.; Ban, H.; Yamaguchi, M. Novel decomplexation method for alkyne- $\mathrm{Co}_{2}(\mathrm{CO})_{6}$ complexes. J. Organomet. Chem. 1998, $554,163-166$.

(20) Wang, M.; Vicente, M. G. H.; Mason, D.; Bobadova-Parvanova, P. Stability of a series of BODIPYs in acidic conditions: an experimental and computational study into the role of the substituents at boron. ACS Omega 2018, 3, 5502-5510.

(21) Kolb, H. C.; Finn, M. G.; Sharpless, K. B. Click chemistry: Diverse chemical function from a few good reactions. Angew. Chem., Int. Ed. 2001, 40, 2004-2021.

(22) Meldal, M.; Tornoe, C. W. Cu-catalyzed azide-alkyne cycloaddition. Chem. Rev. 2008, 108, 2952-3015.

(23) del Rio, M.; Lobo, F.; Lopez, J. C.; Oliden, A.; Bañuelos, J.; Lopez-Arbeloa, I.; Garcia-Moreno, I.; Gomez, A. M. One-pot synthesis of rotationally restricted, conjugatable, BODIPY derivatives from phthalides. J. Org. Chem. 2017, 82, 1240-1247. 
(24) Ziessel, R.; Harriman, A. Artificial light-harvesting antennae: Electronic energy transfer by way of molecular funnels. Chem. Commun. 2011, 47, 611-631.

(25) Avellanal-Zaballa, E.; Ventura, J.; Gartzia-Rivero, L.; Bañuelos, J.; Garcia-Moreno, I.; Uriel, C.; Gomez, A. M.; Lopez, J. C. Towards efficient and photostable red-emitting photonic materials based on symmetric all-BODIPY-triads, -pentads, and -hexads. Chem. - Eur. J. 2019, 25, 14959-14971.

(26) Kaufman, N. E. M.; Meng, Q.; Griffin, K. E.; Singh, S. S.; Dahal, A.; Zhou, Z.; Fronczek, F. R.; Mathis, J. M.; Jois, S. D.; Vicente, M. G. $\mathrm{H}$. Synthesis, characterization, and evaluation of near-IR boron dipyrromethene bioconjugates for labeling of adenocarcinomas by selectively targeting the epidermal growth factor receptor. J. Med. Chem. 2019, 62, 3323-3335. 